\title{
Some Properties of Orthogonal Polynomials
}

\author{
By D. B. Hunter
}

Abstract. Some results are obtained concerning the signs of the coefficients in the expansions in powers of $x^{-1},(1+x)^{-1}$ or $(1-x)^{-1}$ of $1 / p_{n}(x)$ and $q_{n}(x)$, where $p_{n}(x)$ is the polynomial of degree $n$ in the orthogonal sequence associated with a given weight-function $w(x)$ over $(-1,1)$ and $q_{n}(x)=\int_{-1}^{1} w(t) p_{n}(t)(x-t)^{-1} d t$.

1. Origin of the Problem. The problem to be considered here has its origin in some results obtained by Stenger [5]. Let a weight-function $w(x)$ be positive and continuous in the interval $-1<x<1$, and such that $\int_{-1}^{1} w(x) d x$ exists. Then it is well known that there is a sequence of polynomials $\left\{p_{0}(x), p_{1}(x), \cdots\right\}, p_{n}(x)$ being of exact degree $n$, satisfying the orthogonality-relation

$$
\int_{-1}^{1} w(x) p_{m}(x) p_{n}(x) d x=0 \quad(m \neq n)
$$

(see, e.g., Szego [7]). Each polynomial in the sequence is unique apart from a constant factor. We shall impose no particular normalisation on the polynomials, but shall merely stipulate that the coefficient of $x^{n}$ in $p_{n}(x)$ is positive.

A second sequence of functions $\left\{q_{0}(x), q_{1}(x), \cdots\right\}$ can be defined in terms of the above orthogonal sequence by the equation

$$
q_{n}(x)=\int_{-1}^{1} \frac{w(t) p_{n}(t) d t}{x-t}
$$

$q_{n}(x)$ is then analytic and single-valued in the complex plane cut along the interval $[-1,1]$.

The two functions $p_{n}(x)$ and $q_{n}(x)$ have been widely used in recent years in analysing the error in the Gaussian quadrature formulae for integrals of the form $\int_{-1}^{1} w(x) f(x) d x$; among many references, we may mention Barrett [1], Donaldson and Elliott [2], Stenger [5]. Stenger's analysis is concerned largely with the signs of the coefficients $b_{n, j}$ and $c_{n, j}$ in the following two series, which both converge absolutely and uniformly for $|x| \geqslant R>1$ :

$$
1 / p_{n}(x)=\sum_{j=0}^{\infty} b_{n, j} x^{-n-j} \quad(n \geqslant 1)
$$

Received August 6, 1973.

AMS (MOS) subject classifications (1970). Primary 42A52; Secondary 41A55, 33A65.

Key words and phrases. Orthogonal polynomials, weight-function, power-series, Jacobi polynomials, Gaussian quadrature. 
and

$$
q_{n}(x)=\sum_{j=0}^{\infty} c_{n, j} x^{-n-j-1} \quad(n \geqslant 0)
$$

In particular, he shows that if $w(x)$ is an even function of $x$, then

(a) $b_{n, 2 j}>0$ and $b_{n, 2 j+1}=0$, except in the case $n=1$, when $b_{1,0}>0$ and $b_{1, j}=0$ for $j>0$;

(b) $c_{n, 2 j}>0$ and $c_{n, 2 j+1}=0$.

These results are, in fact, quite easily proved. The problem of determining the signs of the coefficients $b_{n, j}$ and $c_{n, j}$ when $w(x)$ is not an even function appears to be considerably more difficult. In Section 2 , we prove two theorems which go part of the way towards solving the problem.

The functions $1 / p_{n}(x)$ and $q_{n}(x)$ can also be expanded in negative powers of $(1+x)$ or $(1-x)$. The corresponding problem for those expansions can be completely solved, and the results are given in Section 3. Section 4 deals briefly with the important special case $w(x)=(1-x)^{\alpha}(1+x)^{\beta}, \quad(\alpha, \beta>-1)$, associated with the Jacobi polynomials $P_{n}^{(\alpha, \beta)}(x)$. Finally, in Section 5 , a number of further results are conjectured.

\section{Expansions in Negative Powers of $x$.}

THEOREM 1. If $w(x) / w(-x)$ is strictly increasing for $-1<x<1$, then $b_{n, j}>0$, $n=1,2, \cdots, j=0,1,2, \cdots$.

Proof. Let the zeros of $p_{n}(x)$ be $x_{1}, x_{2}, \cdots, x_{n}$. It is well known (see, e.g., Szego [7, Theorem 3.3.1]) that they are real and distinct and lie in the open interval $(-1,1)$. We shall arrange them in descending order, so that $x_{1}>x_{2}>\cdots>x_{n}$. Now if $k_{n}$ denotes the coefficient of $x^{n}$ in $p_{n}(x)$, so that $k_{n}>0$, we have

$$
1 / p_{n}(x)=k_{n}^{-1} x^{-n} \prod_{k=1}^{n}\left(1-x_{k} / x\right)^{-1}=k_{n}^{-1} \sum_{j=0}^{\infty} h_{j} x^{-n-j},
$$

where $h_{j} \equiv h_{j}\left(x_{1}, x_{2}, \cdots, x_{n}\right)$ denotes the homogeneous product sum of degree $j$ of $x_{1}, x_{2}, \cdots, x_{n}$ (see, e.g., Littlewood [4, eq. 5.2]). Thus $b_{n, j}=h_{j} / k_{n}$, and it remains to show that $h_{j}>0$.

Now let

$$
w(x, \tau)=\tau w(x)+(1-\tau) w(-x) \quad(0 \leqslant \tau \leqslant 1)
$$

so that, in particular, $w(x, 1)=w(x)$ and $w(x, 0)=w(-x)$. Further, let the zeros of the polynomial of degree $n$ in the orthogonal sequence associated with weight-function $w(x, \tau)$ be $x_{1}(\tau)>x_{2}(\tau)>\cdots>x_{n}(\tau)$.

We have

$$
\frac{w_{\tau}(x, \tau)}{w(x, \tau)}=\frac{w(x)-w(-x)}{\tau w(x)+(1-\tau) w(-x)}=\tau^{-1}-\frac{\tau^{-1}}{w(x) / w(-x)-1+\tau^{-1}},
$$


and this, under the conditions of the theorem, is a strictly increasing function of $x$. Hence, by a theorem of Markoff, (see Szegö [7, Theorem 6.12.1]), the $k$ th zero $x_{k}(\tau)$ is an increasing function of $\tau$. Now clearly, $w(x, 1 / 2)$ is an even function of $x$; consequently, $x_{k}(1 / 2)+x_{n-k+1}(1 / 2)=0$.

Hence

$$
x_{k}+x_{n-k+1}=x_{k}(1)+x_{n-k+1}(1)>0 .
$$

It follows that for $r \geqslant 0, x_{k}^{r}+x_{n-k+1}^{r}>0$.

Thus, if

$$
S_{r}=\sum_{k=1}^{n} x_{k}^{r}
$$

then $S_{r}>0$. But the functions $h_{j}$ are expressible in terms of the $S_{r}$ :

$$
h_{j}=\sum_{(\alpha)} \frac{1}{\alpha_{1} ! \alpha_{2} ! \cdots \alpha_{j} !}\left(\frac{S_{1}}{1}\right)^{\alpha_{1}}\left(\frac{S_{2}}{2}\right)^{\alpha_{2}} \cdots\left(\frac{S_{j}}{j}\right)^{\alpha_{j}}
$$

(see, e.g., Littlewood [4, p. 267]), the summation being over all partitions $(\alpha)=$ $\left(1^{\alpha_{1}} 2^{\alpha_{2}} \cdots j^{\alpha_{j}}\right)$ of $j$. So, clearly, $h_{j}>0$, proving the theorem.

COROllaRY. If $w(x) / w(-x)$ is strictly decreasing for $-1<x<1$, then $(-1)^{j} b_{n, j}>0, n=1,2, \cdots, j=0,1,2, \cdots$.

THEOREM 2. $c_{n, 2 j}>0, n=0,1,2, \cdots, j=0,1,2, \cdots$.

Proof. By expanding $(x-t)^{-1}$ as a power-series in $t / x$, inserting in (2), and integrating term-by-term, we deduce that

$$
c_{n, j}=\int_{-1}^{1} w(t) p_{n}(t) t^{n+j} d t
$$

Now, according to Hildebrand [3, Section 7.4], there is a function $U_{n}(x)$ with the following properties:

$$
\begin{gathered}
w(x) p_{n}(x)=\frac{d^{n}}{d x^{n}} U_{n}(x) \quad(-1<x<1), \\
U_{n}(-1)=U_{n}^{\prime}(-1)=\cdots=U_{n}^{(n-1)}(-1)=0 \\
U_{n}(1)=U_{n}^{\prime}(1)=\cdots=U_{n}^{(n-1)}(1)=0 .
\end{gathered}
$$

Integrating (9) by parts $n$ times and using (10), we thus obtain the result

$$
c_{n, j}=\frac{(n+j) !}{j !} \int_{-1}^{1}(-1)^{n} U_{n}(x) x^{j} d x
$$


We now show that $(-1)^{n} U_{n}(x)>0$ if $-1<x<1$. For $U_{n}^{(n)}(x)\left(\equiv w(x) p_{n}(x)\right)$ has $n$ real zeros in $(-1,1)$, i.e., $U_{n}^{(n-1)}(x)$ has $n$ stationary points in the interval. It follows that $U_{n}^{(n-1)}(x)$ has at most $n+1$ zeros in the closed interval $[-1,1]$. Since two of these are accounted for by the zeros at $x= \pm 1, U_{n}^{(n-1)}(x)$ has at most $n-1$ zeros in $(-1,1)$. Similarly, $U_{n}^{(n-2)}(x)$ has at most $n-2$ zeros in $(-1,1)$, and so on, until, eventually, we see that $U_{n}(x)$ is of constant sign in $(-1,1)$. To establish the sign, we note that

$$
\begin{aligned}
\int_{-1}^{1}(-1)^{n} U_{n}(x) d x & =c_{n, 0} / n !=\frac{1}{n !} \int_{-1}^{1} w(x) p_{n}(x) x^{n} d x \\
& =\frac{1}{n ! k_{n}} \int_{-1}^{1} w(x)\left\{p_{n}(x)\right\}^{2} d x>0
\end{aligned}
$$

so that $(-1)^{n} U_{n}(x)>0$. Thus, if $j$ is even, the integrand in (11) is positive, and this completes our proof.

3. Expansions in Negative Powers of $(1+x)$ or $(1-x)$. The functions $1 / p_{n}(x)$ and $q_{n}(x)$ can also be expanded in negative powers of $(1+x)$ or $(1-x)$, as follows:

$$
\begin{aligned}
1 / p_{n}(x) & =\sum_{j=0}^{\infty} \beta_{n, j}(1+x)^{-n-j} \quad(n \geqslant 1) \\
& =\sum_{j=0}^{\infty} \beta_{n, j}^{\prime}(1-x)^{-n-j} \quad(n \geqslant 1), \\
q_{n}(x) & =\sum_{j=0}^{\infty} \gamma_{n, j}(1+x)^{-n-j-1} \quad(n \geqslant 0) \\
& =\sum_{j=0}^{\infty} \gamma_{n, j}^{\prime}(1-x)^{-n-j-1} \quad(n \geqslant 0) .
\end{aligned}
$$

The two expansions in powers of $(1+x)^{-1}$ are absolutely and uniformly convergent if $|1+x| \geqslant R>2$, those in powers of $(1-x)^{-1}$ if $|1-x| \geqslant R>2$.

The problem of determining the signs of the coefficients $\beta_{n, j}, \beta_{n, j}^{\prime}, \gamma_{n, j}$ and $\gamma_{n, j}^{\prime}$ can be solved completely. The results are stated below, without proof, since the proofs are similar to those of Theorems 1 and 2 (and, in fact, rather simpler).

THEOREM 3.

$$
\left.\begin{array}{r}
\beta_{n, j}>0 \\
(-1)^{n} \beta_{n, j}^{\prime}>0
\end{array}\right\} n=1,2, \cdots, j=0,1,2, \cdots
$$

THEOREM 4. 


$$
\left.\begin{array}{r}
\gamma_{n, j}>0 \\
(-1)^{n+1} \gamma_{n, j}^{\prime}>0
\end{array}\right\} \quad n=0,1,2, \cdots, j=0,1,2, \cdots
$$

4. Application to the Jacobi Polynomials. Let

$$
w(x)=(1-x)^{\alpha}(1+x)^{\beta} \quad(\alpha, \beta>-1) .
$$

Then, apart possibly from a scale-factor,

$$
p_{n}(x)=P_{n}^{(\alpha, \beta)}(x)
$$

the Jacobi polynomial of degree $n$ associated with $w(x)$. Also, $q_{n}(x)$ is closely related to the Jacobi function of the second kind, $Q_{n}^{(\alpha, \beta)}(x)$; in fact, from Szegö [7, Eq. (4.61.4)],

$$
q_{n}(x)=2(x-1)^{\alpha}(x+1)^{\beta} Q_{n}^{(\alpha, \beta)}(x) .
$$

If $\alpha=\beta, w(x)$ is an even function, so that Stenger's results quoted in Section 1 apply. As for the other cases, if $\alpha<\beta$, then $w(x) / w(-x)$ is increasing, so that, by Theorem 1 , $b_{n, j}>0$, while if $\alpha>\beta, w(x) / w(-x)$ is decreasing, and the coefficients $b_{n, j}$ alternate in sign.

In fact, in this case, we can also determine the signs of the coefficients $c_{n, 2 j+1}$, since there is an explicit expression for the function $U_{n}(x)$ of Eq. (10), namely,

$$
U_{n}(x)=\left((-1)^{n} / 2^{n} n !\right)(1-x)^{n+\alpha}(1+x)^{n+\beta}
$$

this corresponds to Rodrigues' formula, see Szegö [7, Eq. (4.3.1)]. So, from (11),

$$
c_{n, 2 j+1}=\frac{(n+2 j+1) !}{2^{n} n !(2 j+1) !} \int_{-1}^{1} x^{2 j+1}(1-x)^{n+\alpha}(1+x)^{n+\beta} d x,
$$

which is positive or negative according as $\alpha<\beta$ or $\alpha>\beta$.

Thus the signs of $b_{n, j}$ and $c_{n, j}$ are completely determined in this case. The expansion of $Q_{n}^{(\alpha, \beta)}(x)$ in powers of $(1-x)^{-1}$ is given in Szegö [7, (4.61.5)] .

5. Some Conjectures. The results proved in the last two sections suggest two further problems:

(a) Can we say anything about the sign of $b_{n, 2 j}$ for a general weight-function $w(x)$ ?

(b) Under what circumstances can we guarantee that $c_{n, 2 j+1}>0$ ?

As to the first problem, it may be conjectured that $b_{n, 2 j}>0$. This would follow immediately if the following purely algebraic conjecture could be proved: 
Conjecture. $h_{2 j}\left(x_{1}, x_{2}, \cdots, x_{n}\right)$ is positive definite for real $x_{1}, x_{2}, \cdots, x_{n}$.

Some related problems are dealt with in Szegö [6]. I have been able to prove the result only in the following special cases:

(i) $j=1$ (all values of $n$ ).

(ii) $n \leqslant 3$ (all values of $j$ ).

(iii) $j=2, n \leqslant 10$.

The proofs in these cases are outlined below.

(i) When $j=1$, Eq. (8) becomes $h_{2}=1 / 2\left(S_{2}+S_{1}^{2}\right)$, which is clearly positive definite.

(ii) The result is obvious when $n=1$. When $n=2$, we have

$$
h_{2 j}\left(x_{1}, x_{2}\right)=\left(x_{1}^{2 j+1}-x_{2}^{2 j+1}\right) /\left(x_{1}-x_{2}\right)
$$

and this is positive, since the numerator and denominator have the same sign.

The case $n=3$ is more difficult. If the three variables have the same sign, $h_{2 j}$ is clearly positive. So we may assume that, say, $x_{1} \geqslant x_{2}>0>x_{3}$. Further, if $x_{1}+x_{3} \geqslant 0$, it follows from the final part of the proof of Theorem 1 that $h_{2 j}$ is positive. So there remains the case $\left|x_{3}\right|>\left|x_{1}\right|$.

From Littlewood [4, Chapter VI, Theorem V],

$$
\begin{aligned}
h_{2 j}\left(x_{1}, x_{2}, x_{3}\right) & =\left|\begin{array}{lll}
x_{1}^{2 j+2} & x_{1} & 1 \\
x_{2}^{2 j+2} & x_{2} & 1 \\
x_{3}^{2 j+2} & x_{3} & 1
\end{array}\right||| \begin{array}{lll}
x_{1}^{2} & x_{1} & 1 \\
x_{2}^{2} & x_{2} & 1 \\
x_{3}^{2} & x_{3} & 1
\end{array} \mid \\
& =\frac{\left(x_{1}-x_{3}\right)\left(x_{1}^{2 j+2}-x_{2}^{2 j+2}\right)+\left(x_{1}-x_{2}\right)\left(x_{3}^{2 j+2}-x_{1}^{2 j+2}\right)}{\left(x_{1}-x_{2}\right)\left(x_{1}-x_{3}\right)\left(x_{2}-x_{3}\right)} \\
& >0 .
\end{aligned}
$$

(iii) The detailed argument in the case $j=2$ is rather involved, and only a brief summary is given. When $j=2$, Eq. ( 8 ) becomes

$$
h_{4}=\frac{1}{24}\left(6 S_{4}+8 S_{3} S_{1}+3 S_{2}^{2}+6 S_{2} S_{1}^{2}+S_{1}^{4}\right) .
$$

The only term which can be negative is that involving $S_{3} S_{1}$. We shall now impose the constraint

$$
S_{2}=1
$$

The minimum value of $S_{4}\left(x_{1}, x_{2}, \cdots, x_{n}\right)$ subject to this constraint is $1 / n$. Hence 


$$
h_{4} \geqslant \frac{1}{24}\left(6 / n+8 S_{3} S_{1}+3\right) .
$$

So, if we can show that the minimum value of $6 / n+8 S_{3} S_{1}+3$ subject to (20) is positive, this proves the result. The problem can be tackled by using Lagrange multipliers.

Unfortunately, a separate argument is required for each value of $n$. It turns out that the required minimum is positive for $n \leqslant 10$; for example, when $n=4$, there is a minimum value $6 / 4+8 S_{3} S_{1}+3=3.677 \cdots$ when

$$
x_{1}=x_{2}=x_{3}=\frac{1}{\sqrt{3}} \sin \frac{7 \pi}{9}, \quad x_{4}=\cos \frac{7 \pi}{9},
$$

and for 7 other sets of values of the variables, obtained by reversing the sign of all of them and/or permuting them. All the other stationary values when $n=4$ correspond to positive values of $S_{3} S_{1}$.

The argument fails when $n=11$; then there is a stationary point for which $6 / 11+8 S_{3} S_{1}+3$ is negative.

In principle, it would be possible, of course, to minimise $h_{4}$ itself rather than $6 / n+8 S_{3} S_{1}+3$, but the algebra then becomes very involved.

As to problem (b), the example of the Jacobi weight-function $w(x)=$ $(1-x)^{\alpha}(1+x)^{\beta}$ suggests that $c_{n, 2 j+1}>0$ if $w(x) / w(-x)$ is strictly increasing, but again no proof has been found.

6. Acknowledgement. I am grateful to the referee for pointing out to me the results contained in Section 4.

School of Mathematics

University of Bradford

Yorkshire, England

1. W. BARRETT, "Convergence properties of Gaussian quadrature formulae," Comput. J., v. 3, 1960/61, pp. 272-277. MR 23 \#1117.

2. J. D. DONALDSON \& D. ELLIOTT," A unified approach to quadrature rules with asymptotic estimates of their remainders," SIAM J. Numer. Anal., v. 9, 1972, pp. 573-602. MR 47 \#6069.

3. F. B. HILDEBRAND, Introduction to Numerical Analysis, McGraw-Hill, New York, 1956. MR 17, 788.

4. D. E. LITTLEWOOD, A University Algebra, 2nd ed., Heinemann, London, 1958. MR 13, 523.

5. F. STENGER, "Bounds on the error of Gauss-type quadratures," Numer. Math., v. 8, 1966, pp. 150-160. MR 33 \#5120.

6. G. SZEGQ̈ "Über gewisse Potenzreihen mit lauter positiven Koeffizienten," Math. Z., v. 37, 1933, pp. $674-688$.

7. G. SZEGÖ, Orthogonal Polynomials, 3rd ed., Amer. Math. Soc. Colloq. Publ., vol. 23, Amer. Math. Soc., Providence, R.I., 1967. 